\title{
REVUE
}

\section{Méthodes et techniques de la chimiothérapie}

\author{
par R. VUILLAUME
}

Le terme "chimiothérapie 》, dont la création est due à Ehrlich, signifiait dans son esprit " une scicnce qui traite de l'action et du mécanisme des substances chimiques sur les cellules, et spécialement des médicaments sur les organismes qui engendrent la maladie $"$.

Cette notion a quelque peu évolué et tend actuellement a designer plus spécialement la destruction des parasites dans le corps même du sujet infesté ce qui implique une spécificité d'action du médicament vis-a-vis du parasite, et une relative innocuité vis-à-vis de l'organisme parasité.

Nous pouvons donc définir la chimiothérapie comme une méthode thérapeutique ayant pour objet la destruction, au sein d'un organisme superieur, des parasites pathogènes (macroscopiques, microscopiques ou ultra-microscopiques) à l'aide de d'investigation et de travail dont nous nous proposons d'exposer l'essentiel.

\section{I. - La découverte de substance's chimiques thérapentiquement actives.}

Cette découverte est empirique, accidentelle ou raisonnée.

Empiriquement, les indiens de l'Amérique du Sud connaissaient depuis fort longtemps, semble-t-il, les propriétés antipaludiques de l'écorce de quinquina. C'est en se basant sur cette observation qu'à partir de la drogue (qui avait été introduite en Europe au XVII siecle), Pelletier, et Caventou isolèrent, en 1820, le principe actif le plus abondant, la quinine.

Chacun sait comment, en 1929, Fleming constata

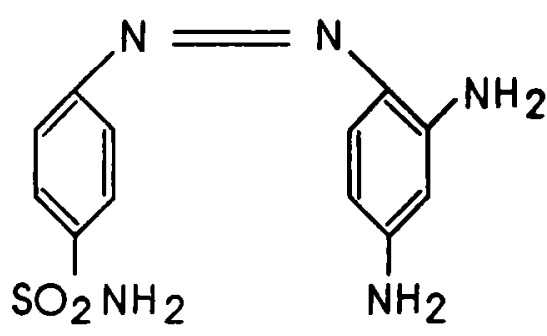

Sulfachrysoidine

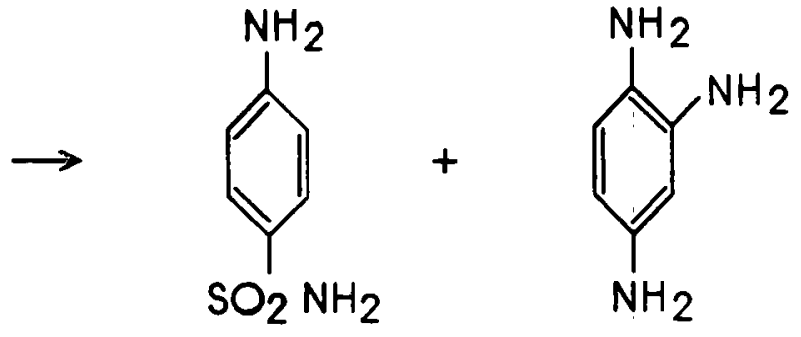

Sulfanilamide
Triaminobenzène

Tableau 1.

substances chimiques ayant une action parasiticide spécifique.

Malgré la diversité des aspects qu'implique une telle définition (qui englobe aussi bien la lutte contre des helminthes que celle contre les ultra-virus), il existe cependant, en matière de chimiothérapie, des règles génórales, des techniques communcs qu'une culture de staphylocoque doré accidentellement souillée par une moisissure, présentait autour de chaque colonie de moisissure une zone circulaire de lyse des colonies staphylococciques. Le génie de Fleming fut de saisir toute l'importance de ce phenomene et de l'attribuer à une substance sćcrétćc par la moisissure. Celle ci ayant été 
identifiée au Penicillium notatum, la substance fut appelée "penicilline » et isolée en 1941.

Dans ces cas, il y a donc, à l'origine, des faits expérimentaux qui dévoilent l'activité thérapeutique d'une substance plus ou moins complexe (écorce de quinquina, jus de culture de $P$, notatum), puis un travail, souvent considérable, de la part de chimistes qualifiés qui, en se basant sur un test d'activité convenable (action fébrifuge de la quinine, action antistaphylococcique de la pénicilline) tentent d'isoler le principe actif à l'état de pureté.

Mais il n'en est pas toujours ainsi et, de plus en plus, les découvertes en chimiothérapie sont le fruit d'investigations raisonnées. Ces investigations sont basées sur cette remarque extrêmement générale qu'il existe une relation entre la structure chimique des composés organiques et leur action sur les êtres vivants. Deux manières d'opérer sont possibles :

a) On peut essayer, contre un parasite, une substance déjà connue pour son activité contre un autre parasite plus ou moins voisin du premier. C'est ainsi que, connaissant l'action de certains colorants azoïques sur des protozoaires, Domagk, en 1935, fut amené à étudier l'action de ces mêmes colorants vis-à-vis des streptocoques dans l'infection expćrimentale de la Souris. Il découvrit alors la haute activité antistreptococcique de la sulfachrysoildine, activité que, tout naturellement, il attribua d̀ la foriction azoïque. Puis en 1936, Trefouël et ses collaborateurs montrèrent que cette explication était erronée, que l'azoïque était coupé dans l'organisme et que, sur les deux corps qui prenaient ainsi naissance, seul le sulfanilamide (1.162 F.) était antistreptococcique (tableau 1).

b) On peut encore, lorsqu'on connaît déjà une substance active vis-à-vis d'un parasite, préparer par synthèse toute unc sćric do substances de structure voisine et essayer leur activité. Par exemple, la découverte de l'activité antistreptococcique du sulfanilamide a été à l'origine de la préparation et de l'emploi de toute la gamme des " médicaments sulfamidés » qui en dérivent par substitution.

De même, la connaissance de la formule développée de la quinine (établie vers 1920) fut à l'origine des travaux qui devaient aboutir à la préparation et à la découverte des principaux antipaludeens de synthèse actuellement utilisés : Pamaquine, Mépacrine (1929), Chloroquine (1941), Proguanide (1946); le tableau 2 montre la parenté structurale qui existe entre chacune de ces substances et la précédente ou la suivante, bien que, en définitive, la constitution du Proguanide soit fort éloignée de celle de la quinine.
Tableau 2.

Antipaludéens ( $\mathrm{R}, \mathrm{R}$, $\mathrm{R}^{\prime \prime}$ =' radicaux organiques).<smiles>[R]c1ccnc2ccc(OC)cc12</smiles><smiles>[R]Nc1cc(OC)cc2c1NCC=C2</smiles><smiles>[R]NC12CN(c3cc(Cl)ccc31)c1ccc(OC)cc12</smiles><smiles>[R]c1ccnc2cc(Cl)ccc12</smiles><smiles>[R]Nc1ccnc(Nc2ccc(Cl)cc2)n1</smiles><smiles>[R]NC(=N)NC(=N)Nc1ccc(Cl)cc1</smiles>

(Substance intermédiaire, douée d'activité) Proguanide 
Lorsque, d'une manière ou d'une autre, une substance active (ou qu'on suppose active) a été découverte, elle est soumise à l'étude experimentale.

\section{Étude expérimentale d'une substance chimique en vue de son emploi en chimiothérapie.}

Cette étude comporte essentiellement la recherche de la toxicité (détermination de la dose mortelle) pour les organismes qui hébergent le parasite: la recherche de l'activité thérapeutique (détermination de la dose curative): enfin, l'étude du métabolisme de la substance et notamment de sa vitesse de disparition dans l'organisme.

\section{2) Étude de la toxicité.}

La nécessite de connaitre la toxicité des substances utilisables en chimiothérapie est évidente : Primum non nocere. Ce qui importe, c'est évidemment la toxicite pour l'espece animale que l'on veut traiter; mais lorsqu'il s'agit de grands animaux, on peut réduire les frais d'expérimentation en opérant d'abord sur des petits animaux de laboratoire (Souris, Rat, Cobaye, Lapin, Chien, Chat, Poulet), et en transposant ensuite les resultats, sous les réserves que nous préciserons plus loin.

La toxicité d'une substance peut être définie par la dose minima mortelle (= dose léthale, ou D.L.), c'est-à-dire la plus petite dose qui provoque la mort de l'animal considéré. La détermination de la D.L. n'est pas simple, car elle varie avec beaucoup de facteurs, notamment avec l'espèce animale, le poids du sujct, la voie d'introduction, l'état de santé.

Tous ces facteurs étant rendus identiques, on peut encore observer des variations très importantes, allant en moyeme du simple au double, suivant les individus. C'est-à-dire que si l'on prend deux animaux de même espèce, de même race, de même àge, de même sexe, de mème poids, recevant la même substance administrée de la même façon, on pourra observer la mort de l'un des animaux avec la moitié d'une dose qui pourra être supportee par l'autre.

Ces variations individuelles ont eté traduites graphiquement par Trewan sous la forme d'une courbe représentative du pourcentage de mortalité en fonction de la dose administrée (dans des conditions expérimentales definies). Quels que soient les substances et les animaux, on obtient toujours une courbe en forme d'S (plus cu moins incline), représentée tableau 3.

De telles courbes peuvent être construites point par point, en administrant les doses figurant en abcisses à des lots d'animaux et en notant le pourcentage de mortalité dans chaque lot. D'après ce qui précède, chaque lot doit comporter un nombre suffisant daanimaux afin d'éliminer des variations individuelles (au moins 30 par lot).

Les courbes de mortalité permettent alors de definir la dose mortelle moyenne, ou dose léthale moyenre (D.L. 50) comme la dose qui, dans les conditions de l'expérimentation, provoque la mort de la moitié des animaux. La détermination de cette D.L. 50 necessite donc la mise en cuvre d'un assez grand nombre d'animaux; on peut cependant avoir reccurs a des méthodes simplifiées; certaines ont

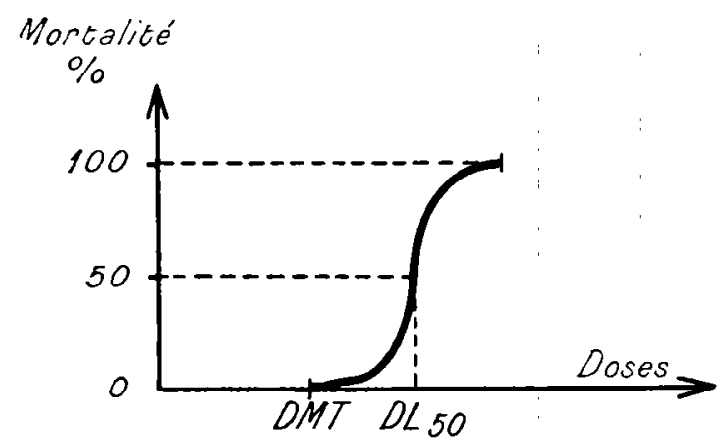

ete proposées qui permettent, avec une approximation satisfaisante, de déduire la D.L. 50 de la determination de deux points seulement de la courbe.

Les courbes de mortalité permettent encore de définir la dose maxima tolérée (ou D.M.T.) qui est la dose la plus forte qui, dans les conditions de l'expérimentation, peut être administrée sans risque de provoquer la mort (= dose mortelle $0 \%$ ). On voit immédiatement l'importance que peut présenter la D.M.T. en chimiotherapie.

F. côté des influences individuelles; des facteurs importants (pour la chimiothérapic) de variation de la dose mortelle sont l'espèce animale et le poids des sujets. Il est difficile de séparer ces deux facteurs, car les variations dues à l'espèce sont généralement la conséquence d'une différence de poids, et, lorsqu'il n'en est pas ainsi, il est obligatoire d'éliminer l'influence du facteur "poids » pour discerner la part qui revient au facteur « espèce ").

Par exemple, dire que la D.M.T. de tryparsamide, par voie intraveineuse, est de :

2 grammes par kilo chez la Souris;

o gr. 75 par kilo chez le Lapin,

ne signifie pas, à priori, que la sensibilité du Lapin soit supérieure à celle de la Souris. Les poids moyens de ces animaux étant différents, la conclusion précédente ne serait valable qu'autant qu'on admettrait d'abord que, dans une même espèce, la dose à administrer chez un animal pour obtenir un effet donné doit être proportionnelle à son poids. Or, de nombreuses expériences ont montré, au contraire, que la dose $D$ n'est nullement proportionnelle au 
poids $\mathrm{M}$ de l'animal, mais bien à la puissance $2 / 3 \mathrm{du}$ poids (Puissance $2 / 3=$ racine cubique du carré) :

$$
\mathrm{D}=k \mathrm{M}^{2 / 3}
$$

ce qui peut s'écrire (D, D', $D^{\prime \prime}$... étant les doses correspondant à des animaux de poids $M, M^{\prime}$, $M^{\prime \prime}, \ldots$ ) :

$$
\begin{gathered}
\frac{D}{M^{2 / 3}}=\frac{D^{\prime}}{M^{\prime 2 / 3}}=\frac{D^{\prime \prime}}{M^{\prime \prime 2} / 3}=\ldots=k \\
\text { d'où : } \frac{D}{D^{\prime}}=\left(\frac{M}{M^{i}}\right)^{2 / 3}
\end{gathered}
$$

Cette formule qui, théoriquement, n'est valable que pour les animaux d'une même espèce, peut pratiquement être appliquée à des animaux d'espèces différentes, au moins en première approximation, ou pour juger de la sensibilité d'espèce et faire des comparaisons. Ainsi, en reprenant l'exemple cité plus haut : la D.M.T. de tryparsamide par voie intraveineuse pour la Souris étant de 2 grammes par kilo, soit $D^{\prime}=4$ centigrammes pour une souris de poids $M^{\prime}=20$ grammes, si nous admettons que le Lapin présente la même sensibilité que la souris, la D.M.T. (D) pour un Lapin de poids $\mathrm{M}=2$ kilos serait :

$$
\mathrm{D}=\mathrm{D}^{\prime}\left(\frac{\mathrm{M}}{\mathrm{M}^{\mathrm{i}}}\right)^{2 / 3}=0,04\left(\frac{2.000}{20}\right)^{2 / 3}=0 \text { gr. } 86
$$

soit une D.M.T. calculée pour le Lapin de 0 gr. 43 par kilo. Or, nous avons indiqué plus haut que cette D.M.T. était en réalité de $0 \mathrm{gr} .75$ par kilo. On peut donc conclure que le Lapin est un peu moins sensible au tryparsamide que la Souris, contrairement à ce qu'il apparaissait à première vue.

Par conséquent, lorsqu'on se servira de cette même formule pour transposer les résultats obtenus dans une espèce à une autre espèce animale (et, en particulier, pour transposer les résultats obtenus chez des animaux de laboratoire aux grandes espèces), il importera do sc souvenir que cette opération suppose une absence de différence dans la sensibilité des espèces considérées. En règle générale, cette manière de faire dorne des résultats satisfaisants, mais des exceptions existent, et il sera toujours nécessaire de vérifier le bien fondé de notre raisonnement par quelques sondages expérimentaux.

L'influence de l'état de santé des animaux sur la dose mortelle présente aussi un intérêt : les animaux infectés réagissient souvent moins bien que les animaux sains vis-à-vis des intoxications. Une D.M.T. déterminée sur des animaux sains pourra donc provoquer une certaine mortalité chez des animaux infectés, et cela doit toujours être pris en considération.

Enfin, si, dans un but de simplification, la chimiothéràpie a recours, autant que possible, à des substances qui agissent efficacement à la suite d'une seule administration aux malades, il arrive qu'il soit nécessaire de renouveler les doses de façon à maintenir dans l'organisme pendant un certain temps un certain taux de la substance. Or, si une seule dose inférieure à la D.M.T. ne doit pàs provoquer d'accident mortel, il n'en va plus de même si cette dose est répétée à intervalles de temps plus ou moins rapprochés. Pour compléter nos connaissances sur la toxicité de cette substance, il faudra déterminer expérimentalement la " dose quotidienne tolérée ", c'est-à-dire la plus forte dose qui, administrée quotidiennement pendant un temps donné, n'entraîne aucun accident toxique permanent. Cétte dose est souvent bien inférieure à la D.M.T.

\section{b) Étude de l'activité thérapeutique.}

L'activité thérapeutique peut êlre définie, en chimiothérapie, par la dose curative, c'est-à-dire par la dose capable d'établir, dans l'organisme, pendant un temps suffisant, une, concentration toxique pour le parasite.

Cette definition implique deux ordres de recherches': 'd'une part, la détermination de la concentration toxique pour le parasite, en fonction du temps de contact, d'autre part, l'étude du métabolisme de la substance dans l'organisme qui héberge le parasite, et notamment l'étude de sa vitesse de disparition qui permettra d'établir le mode et, éventuellement, le rythme d'administration de la substance.

Ia dose toxique pour le parasite peut être recherchée directement sur celui-ci, pris en dehors de l'organisme; par exemple sur une culture microbienne : on détermine les concentrations minima et optima assurant la destruction. Cette méthode opératoire in vitro n'est pas, toujours suffisante; l'action ainsi observée n'est pas toujours identique à celle qui se manifeste in vivo. Ainsi la sulfachrysoïdine qui est très fortement antistreptococcique chez la Souris infectée est pratiquement sans action sur une culture de ces mêmes streptocoques. Il en sera ainsi de toutes les substances qui, comme la sulfamide chrysoidine, subissent dans l'organisme une transformation nécessaire à faire apparaître leur activité thérapeutique.

Aussi les meilleures méthodes de détermination de la dose curative consistent-elles à travailler directement sur des animaux parasités (naturellement ou expérimentalement). On opère exactement comme il a été dit pour la determination des doses mortelles, car les causes de variation de la dose curative sont les mêmes. Par exemple, si on représente graphiquement le pourcentage des animaux guéris en fonction de la dose, on obtient 
une courbe ayant la forme des courbes de mortalité, et on appelle dose curative (D.C.) la dose juste nécessaire qui, dans les conditions de l'expérimentation, fait disparaître les parasites chez tous les sujets (= dose stérilisante $100 \%$ ).

Comme pour la dose mortelle, les résultats obtenus pour une espèce animale pourront être transposés dans une autre espèce par application de la formule $\mathrm{D}=k \mathrm{M}^{\mathrm{s} / 3}$, suivie d'une vérification expérimentale.

\section{c) Étude du métabolisme.}

On cherche surtout à connaitre la distribution de la substance dans l'organisme et sa vitesse de disparition; dans certains cas, il y a intérêt à rechercher les produits de transformation, lorsque l'un ou plusieurs d'entre eux sont responsables de l'activité thérapeutique.

La répartition dans les différents tissus et liquides de l'organisme est décelée par des dosages de la substance sur des prélèvements effectués simultanément en divers points de l'organisme. Très souvent, on peut se contenter de déterminer la concentration sanguine et de rechercher l'importance de la dose à administrer pour obtenir une concentration sanguine au moins égale à la concentration efficace sur le parasite.

Mais l'organisme se débarrasse de cette substance qui lui est étrangère, soit par destruction (en mettant en jeu des mécanismes de "détoxication "), soit par élimination (avec l'urine, la bile). De sorte que la concentration sanguine est variable dans le temps.

$\mathrm{Si}$, par des dosages sur des prélevements sanguins répartis dans le temps, on cherche à tracer la courbe

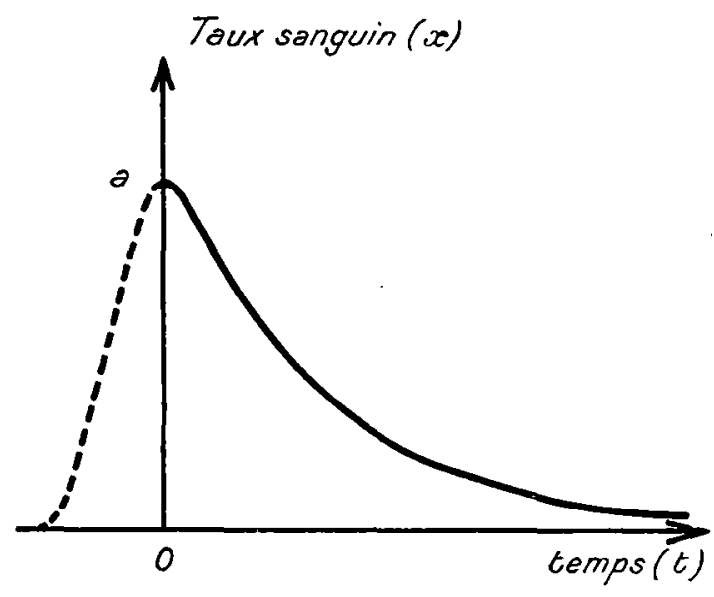

Tableau 4.

représentative de la concentration sanguine $(x)$ en fonction du temps $(t)$, on observe d'abord un accroissement de $x$ jusqu'à un maximum a. Ce maximum est atteint plus ou moins rapidement, suivant la voie d'administration de la substance, sa diffusibilité, etc. ; il est atteint en quelques secondes si l'administration est faite par voie intraveineuse.

A partir de ce maximum, la courbe prend, le plus généralement, une allure exponentielle (tableau 4), c'est-à-dire qu'elle peut être définie par une équation de la forme (en prenant le moment correspondant au maximum a comme origine des temps) :

$$
x=a e^{-k t}
$$

où $e+2,718 \ldots$ et où $k$ est une constante qui dépend à la fois de la substance et de l'animal.

Les valeurs $a$ et $k$ définissent donc entièrement la courbe (1). Si $k$ est grand, la pente de la courbe $\left(d x_{i}^{i} d t=-x\right)$ sera grande : il s'agit d'une substance à disparition rapide. C'est le cas de la pénicilline qui est pratiquement éliminée au bout de quelques heures. $\mathrm{Si}$, au contraire, $k$ est petit, la pente de la courbe sera faible, la substance subsistera longtemps dans l'organisme. C'est le cas des substances dites "à accumulation 》, comme par exemple les arsenicaux, dont l'élimination demande plusieurs semaines.

De telles courbes permettent de savoir au bout de combien de temps la concentration sanguine consécutive à l'administration d'une quantité donnée de substance tombe au-dessous de sa valeur efficace, et de vérifier si ce temps est suffisant pour assurer la destruction des parasites. S'il ne l'est pas, il faudra, ou bien augmenter la dose initiale (si la D.M.T. le permet), ou bien répéter l'administration à des intervalles de temps qu'il sera facile de preciser.

\section{Le choix de la substance la meilleure.}

Lorsque l'étude expérimentale a montré l'efficacité de plusieurs substances vis-à-vis d'une même maladie, il y a lieu de comparer entre eux les avantages et les inconvénients de ces substances, afin de fixer un choix raisonné.

Le premier point qui retiendra l'attention sera évidemment l'activité thérapeutique; mais cette appréciation de l'activité doit tenir compte de la toxicité, car de quelle utilité serait une substance très active si une grande toxicité en interdisait pratiquement l'emploi ?

Un cas simple est celui où l'on doit comparer entre elles des substances présentant sensiblement la même toxicité, comme, par exemple, les substances appartenant à une même série chimique. Il suffit alors de les soumettre à un test d'activité convenablement choisi. Le tableau 5 donne un exemple

(1) On peut encore définir la courbe, ainsi que l'a proposé LAPP en 1948, par l'indication de la vitesse initiale de disparition (protorrhée) et du temps au bout duquel le taux de la substance a diminué de moitié (hémikrèse). 
d'une telle étude, où l'on compare l'activité antimalarique de substances dérivées du diguanide. Le test choisi est la recherche de la dose curative chez le Poulet infesté par Plasmodium gallinaceum. Il est facile de voir que la meilleure des substances examinées est celle qui porte le no 4888.

\section{Tableau 5}

Activité antimalarique des dérivés du diguanide de la forme

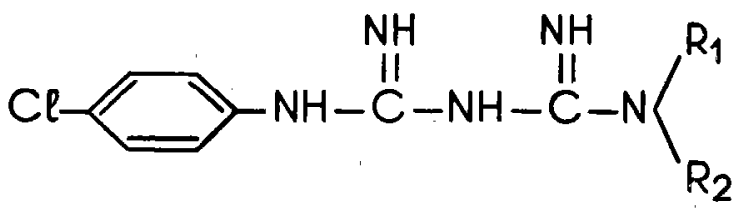

Expérimentation sur le Poulet infesté par Plasmodium gallinaceum. Administration per os.

\begin{tabular}{|c|c|c|c|c|}
\hline $\mathbb{N}^{o}$ & $\mathrm{R}_{1}$ & $\mathrm{R}_{2}$ & $\begin{array}{c}\mathrm{DOSE} \\
\text { en } \mathrm{mg} / \mathrm{kg}\end{array}$ & ACrifté \\
\hline 3327 & $-\mathrm{H}$ & $-\mathrm{H}$ & 125 & - \\
\hline 5093 & $-\mathrm{CH}_{3}$ & $-\mathrm{H}$ & 80 & - \\
\hline 4134 & $-\mathrm{CH}_{3}$ & $-\mathrm{CH}_{3}$ & 80 & - \\
\hline $496 ?$ & $-\mathrm{C}_{2} \mathrm{H}_{5}$ & $-\mathrm{H}$ & 20 & $+t$ \\
\hline 3926 & $-\mathrm{C}_{2} \mathrm{H}_{3}$ & $-\mathrm{C}_{2} \mathrm{H}_{5}$ & 80 & ++ \\
\hline 4887 & $-\mathrm{CH}_{2}-\mathrm{CH}_{3}-\mathrm{CH}_{3}$ & $-\mathrm{H}$ & 20 & $++t$ \\
\hline 4888 & Isopropyl & $-\mathrm{H}$ & 10 & +++ \\
\hline 4329 & Propyl & $-\mathrm{CH}_{3}$ & 40 & ++ \\
\hline 4430 & Isopropyl & $-\mathrm{CH}_{3}$ & 16 & $t+t$ \\
\hline 4968 & $-\mathrm{CH}_{2}-\mathrm{CH}-\mathrm{CH}_{2}$ & $-\mathrm{H}$ & 20 & ++ \\
\hline 4565 & Butyl & $\mathrm{H}$ & 80 & -11 \\
\hline 4567 & Isobutyl & $-H$ & 320 & + \\
\hline 4568 & Isopentyl & $-\mathrm{H}$ & 40 & ++ \\
\hline 4095 & Butyl & Butyl & 400 & + \\
\hline 4635 & Pentyl & $-\mathrm{H}$ & 80 & $+t$ \\
\hline 5234 & $-\mathrm{OCH}_{3}$ & $-\mathrm{H}$ & 80 & - \\
\hline
\end{tabular}

Tableau tiré de Curd (F'S.H.), Davey et Rose (F.L.) :

\section{Ann. Trop. Med. Parasitol, 1945, 39, 208.}

Mais le plus souvent, les substances à comparer ont des toxicités différentes. Il faut alors tenir compte de leur marge de sécurité, c'est-à-dire de l'écart qui existe entre la D.M.T. (dose tolérée) et la D. (dose guérissant $100 \%$ des animaux). Cet écart peut être traduit simplement par le rapport D.M.T.D.C. appelé coefficient thérapeutique : la substance sera d'autant meilleure que son coefficient sera plus élevé, c'est-à-dire que la D.C. sera petite par rapport à la D.M.T.

L'emploi de ce coefficient thérapeutique comme moyen de comparaison appelle quelques remarques : Il ne faut pas oublier que ce coefficient, comme la D.M.T. et la D.C. peut présenter, pour une même substance, des valeurs différentes suivant les conditions expérimentales, et surtout suivant le parasite considéré et l'espèce animale utilisée lors de sa détermination.

Par exemple, le coefficient thérapeutique de la stilbamidine, déterminé par administration intraveineuse chez la Souris infestée par des Trypanosomes, prend les valeurs suivantes:

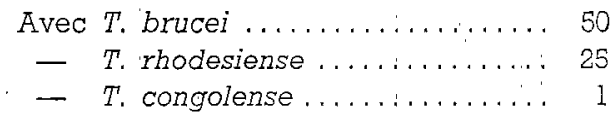

De même, le coefficient de $205 \mathrm{~B}$, déterminé par administration intraveineuse chez des animaux infestés par T. equiperdum prend les valeurs suivantes :

Chez la Souris................... 100

- le Lapin................... 10

- le Cheval .................. 2

Ces écarts considérables soulignent l'extrême importance qu'il y a à ne comparer entre eux que des coefficients thérapeutiques qui ont été établis dans des conditions expérimentales rigoureusement identiques. Il est illusoire de vouloir comparer des coefficients établis par des auteurs différents, et à plus forte raison si on ne sait pas quels sont les tests qui ont été choisis pour établir ces coefficients.

Enfin, dans la comparaison et le choix des substances, on devra également tenir compte de la durée et de la facilité de conservation, du prix de revient du traitement, de la plus ou moins grande facilité d'administration (une seule injection est toujours preférable à plusieurs; une injection souscutanée ou intramusculaire est préférable à une injection intraveineusc), à la frćquence des accidents : à la possibilité d'accoutumance des parasites, etc. 'Tous ces facteurs montrent la' nécessité de toujours compléler l'élude faite au laboratoire par une large expérimentation clinique qui est seule capable, en définitive, de permettre de porter un jugement convenable.

\section{Mise en pratique de la chimiothérapie. Moda-}

\section{lités. Accidents.}

La mise en pratique est soumise à un certain nombre de règles générales que nous rappelleroins brièvement. La chimiothérapie doit être :

- précoce : les résultats sont d'autant meilleurs 
que les agents parasites sont moins nombreux, que l'organisme est moins infesté, plus apte à se défendre:

- massire : l'effet curatif apparaitra plus rapidement si on réalise plus vite dans l'organisme la concentration efficace contre les parasites; l'établissement progressif ou différé de cette concentration efficace riscue d'ailleurs de provoquer une accoutumance cies parasites à la substance; il faut donc, autant que possible, administrer d'emblée une dose suffisante

- soutenie : si une seule administration de la substance est insuffisante, il faut répéter l'opération autant de iois qu'il sera nécessaire pour maintenir dans l'organisme, pendant un temps suffisant, la concentration qui doit assurer la guérison des malades. Lorsqu'une substance à disparition rapide dans l'organisme doit faire l'objet d'administrations répétées, ce qui est pratiquement un gros inconvénient, on ceut en partie tourner la difficulté par l'emploi dune méthode "retard" qui consiste à accroitre la durée du séjour de la substance dans l'organisme, soit en retardant son absorption (emploi d'excipients spéciaux, huileux; de formes peu solubles, microcristallines), soit en retardant son élimination urinaire (associations avec la polyvinylpyrrolicione, le caronamide).

La chimiothérapie n'est pas seulementune méthode curative; elle peut aussi être préventive : les substances chimiques parasiticides peuvent être administrées chez des animaux sains pour les protéger contre une atteinte ultérieure d'agents parasites. Les doses préventives à employer sont déterminées par une expérimentation comparable à celle décrite pour l'étao'issement de la dosc curative. De toule évidence. il faut préférer ici des substances qui persistent longtemps dans l'organisme (substances à cisparition lente, ou emploi de méthodes retard).

Enfin, rappelons que des accidents sont toujours possibles. Ils sont de deux ordres:

Les uns sont des accidents allergiques : certains sujets, particulièrement sensibles ou sensioilisés a la substance, peuvent présenter un choc plus ou moins grave dès l'administration dune dose, même très inférieure à la dose curative usuelle. Ces accidents ne sont pas particuliers à la chimiothérapie; ils sont difficiles à prévoir, mais heureusement fort rares chez les animaux.
Les autres sont d'ordre toxique proprement dit : Iis peuvent se produire dès la première administration: surtout lorsque la dose injectée est très voisine de la D.M.T. : celle-ci ayant été déterminée par une méthode statistique, on peut exceptionnellement rencontrer des sujets qui ne la supportent pas. Les accidents toxiques peuvent aussi se produire à la longue, lors d'administrations répétées de la substance active (therapeutique soutenue), Tel est le cas des accidents d'arsénicisme dus à une médication arsénicale prolongée. Les accidents peuvent aussi être cus à une forme de transformation de la substance dans l'organisme (exemple : cristallisation intrarénale des produits d'acétylation des médicaments sulfamidés); ou encore à des phénomòncs de photoscnsibilisation, lors de l'emploi de substances colorantes (dérivés trypanocides de la phénanthridine).

On peut diminuer la fréquence de ces divers accidents grâce à de judicieuses associations médicamenteuses.

Ainsi, la vitamine $C$, les thiols permettent à l'organisme de mieux résister à l'intoxication arsénicale. Les associations à synergie additive permettront de lutter contre les accidents dus à des produits de transformation des substances introduites dans l'organisme (exemple : association de sulfamides entre eux); enfin et surtout, les associations à synergie renforçatrice (potentialisation) réduiront considerablement les dangers dus à la toxicité propre des substances employées. Comme exemples de ce dernier mode, citons l'association préconisée par Launoy du $309 \mathrm{~F}$ avec les antimoniaux (contre les tryoanosomes) l'association des sulfamides avec certains antibiotiques d'origine fongique.

Ce rapide tour d'horizon n'a d'autre prétention que de rappeler les caractères généraux des méthodes de la chimiothérapie. Nous avons volontairement laissé de côté certains points comme par exemple l'étude du mode d'action des substances sur les parasites; cette action est très variable et malgrè l'intérêt de ces recherches, elles s'écartent dejà des généralités où nous avons volontairement maintenu notre exposé. On voit que la mise au point ces traitements nécessite la collaboration de chercheurs appartenant à des disciplines diverses : chimistes, physiologistes, cliniciens: C'est une méthode relativement récente, puisqu'elle ne date guère que du début du XX $\mathrm{XX}^{\mathbf{e}}$ siècle; c'est une méthode a'avenir. 